TRANSACTIONS OF THE AMERICAN MATHEMATICAL SOCIETY

Volume 348, Number 3, March 1996

\title{
PARTIALLY HYPERBOLIC FIXED POINTS WITH CONSTRAINTS
}

\author{
PATRICK BONCKAERT
}

\begin{abstract}
We investigate the local conjugacy, at a partially hyperbolic fixed point, of a diffeomorphism (vector field) to its normally linear part in the presence of constraints, where the change of variables also must satisfy the constraints. The main result is applied to vector fields respecting a singular foliation, encountered, by F. Dumortier and R. Roussarie, in the desingularization of families of vector fields.
\end{abstract}

\section{INTRODUCTION AND MOTIVATION}

Let $x$ denote the variable in the center manifold near a partially hyperbolic singularity 0 of a vector field $X$ and let $y$ be the normal variable; then we can write $X=X_{x} \frac{\partial}{\partial x}+X_{y} \frac{\partial}{\partial y}$ and define its normally linear part by $N X:=X_{x}(x, 0) \frac{\partial}{\partial x}+$ $\frac{\partial X_{y}}{\partial y}(x, 0) \cdot y \frac{\partial}{\partial y}$. We study the question about conjugating $X$ to $N X$ near the singularity, i.e. about finding new coordinates in which the vector field is normally linear. Remark that $N X$ is linear in its $y$ variable. If there are no further requirements on this new coordinates then there is a theorem of $\mathrm{F}$. Takens $[\mathrm{T}]$ stating that, under certain nonresonance conditions on the eigenvalues at the singularity, one can find $C^{k}$ coordinates near 0 for any $k \in \mathbb{N}$ putting the vector field in normally linear form.

However in many problems extra constraints show up like e.g. the fact that a vector field can be a family of lower dimensional vector fields and that at each stage (normal forms, coordinate changes) we want to preserve this family character. In their paper about a geometric description and explanation of the canard phenomenon [DR2] F. Dumortier and R. Roussarie encounter vector fields respecting a singular foliation and need to use normal forms and coordinate changes respecting this extra structure. More precisely they need the following result that I propose to prove in this paper, as a consequence of a more general theorem. It concerns families of vector fields on $\mathbb{R}^{3}$ having a partially hyperbolic fixed point at the origin and which have $\mathbb{R}^{2} \times\{0\}$ as a center manifold. Let $x=\left(x_{1}, x_{2}\right)$ denote the variables in this center manifold.

Received by the editors March 8, 1994.

1991 Mathematics Subject Classification. Primary 58F14, 58F36, 34C20.

Key words and phrases. Partially hyperbolic fixed point, normal form, constraint, structure preserving.

(C)1996 American Mathematical Society 
Theorem 1. Let $p, q \in \mathbb{N}$, and let $k \in \mathbb{N}$ be given. There exists $N \in \mathbb{N}$ such that the following holds. Let $\left(x_{1}, x_{2}, y\right)$ denote variables of $\mathbb{R}^{3}$ and let $X_{\lambda}=X_{\lambda}\left(x_{1}, x_{2}, y\right)$ be a $C^{N} P$-parameter family of vector fields defined near $0 \in \mathbb{R}^{3}$ having the following properties

(i) $X_{\lambda}$ is tangent to the level surfaces $x_{1}^{p} x_{2}^{q}=$ constant.

(ii) the linear part $d X_{0}(0)$ of $X_{0}$ at the origin has exactly one non-zero eigenvalue and $y=0$ is the related center manifold.

Then there exists a local family of $C^{k}$ changes of variables $H_{\lambda}$ near the origin $\left(\lambda, x_{1}, x_{2}, y\right)=0$ such that in the new coordinates

$$
\begin{aligned}
& \left(H_{\lambda}\right)_{*} X_{\lambda}\left(x_{1}, x_{2}, y\right) \\
& \quad=X_{\lambda, 1}\left(x_{1}, x_{2}, 0\right) \frac{\partial}{\partial x_{1}}+X_{\lambda, 2}\left(x_{1}, x_{2}, 0\right) \frac{\partial}{\partial x_{2}}+\frac{\partial X_{\lambda, 3}}{\partial y}\left(x_{1}, x_{2}, 0\right) \cdot y \frac{\partial}{\partial y}
\end{aligned}
$$

and such that moreover $H_{\lambda}$ leaves the level surfaces $x_{1}^{p} x_{2}^{q}=$ constant invariant.

The vector field like in the last expression is thus the normally linear part of $X_{\lambda}$. So the change of variables $H_{\lambda}$ has to satisfy a certain constraint.

In particular one also asks that a family of vector fields is conjugated to its normally linear part using a family of changes of variables. This is also a type of constraint if we consider the parameter direction as an extra direction in the center manifold: from the theorem of Takens it does not follow immediately that the change of variables $H(\lambda, x, y)$ is equal to $\lambda$ in the parameter direction. As a matter of fact this result about families is often used [BP,CP,IY,NPT,PT], since the adaptations in the proof of $[\mathrm{T}]$ are minor; but it will follow as a trivial application of our methods. Special thanks to Freddy Dumortier and Robert Roussarie for inspiring discussions.

\section{The RESUlT}

We will give the theorem (and its proof) for diffeomorphisms, but, as usual, a similar result holds for vector fields (cf. the adaptations in $[\mathrm{T}]$ ). For the sake of readability of the paper we will assume that the codimension of the centermanifold is equal to one: then there are no nonresonance conditions on the eigenvalues and the essential ideas are maybe more clear.

In order to fix the notations we consider diffeomorphisms $f: \mathbb{R}^{c} \times \mathbb{R} \rightarrow \mathbb{R}^{c} \times \mathbb{R}$ : $(x, y) \mapsto\left(f_{x}, f_{y}\right)(x, y)$ having a partially hyperbolic fixed point at the origin $0 ; \mathbb{R}^{c}$ is the centermanifold. Mostly we will write $v=(x, y) \in \mathbb{R}^{c} \times \mathbb{R}$ and $f=\left(f_{x}, f_{y}\right)$.

We only aim for results on a neighbourhood of $(x, y)=(0,0)$, so everything is local, except for some constructions mentioned further on with cut off functions. All germs are taken at the origin.

Definition. If $f$ is a diffeomorphism of $\mathbb{R}^{c} \times \mathbb{R}$ having $\mathbb{R}^{c}$ as a centermanifold, then we call

$$
N f(x, y)=\left(f_{x}(x, 0), \frac{\partial f_{y}}{\partial y}(x, 0) \cdot y\right)
$$

its normally linear part. 
(2.1) The formal, the semiformal and the path condition. Let $D_{0}$ be a group of germs in $0 \in \mathbb{R}^{c} \times \mathbb{R}$ of diffeomorphisms fixing 0 .

The formal condition. First of all we have a bunch of notations about multilinear maps coming from Taylor developments. Let $i, j \in \mathbb{N}$. If $A$ is an $i+j$-linear operator we will write shortly $A x^{i} y^{j}:=A(x, \ldots, x, y, \ldots, y)(i$ times $x$ and $j$ times $y)$. This will be used in the Taylor development in the origin of a map $f(x, y)$, namely $A=\frac{1}{(i+j) !} \frac{\partial^{i+j} f}{\partial x^{i} \partial y^{j}}(0,0)$. If moreover $L$ and $M$ are linear operators in $x$ resp. $y$ we will abbreviate

$$
A L^{i} M^{j} \cdot x^{i} y^{j}:=A(L x, \ldots, L x, M y, \ldots, M y)
$$

( $i$ times $L x$ and $j$ times $M y$ ). The space of $i+j$-linear maps $\left(\mathbb{R}^{c}\right)^{i} \times \mathbb{R}^{j} \rightarrow \mathbb{R}^{c} \times \mathbb{R}$ will be denoted by $I_{i j}$. Let us denote, for $i, j \in \mathbb{N}$

$$
I_{i j}\left(D_{0}\right)=\left\{C \in I_{i j} \mid \exists H \in D_{0}: H(x, y)=(x, y)+C x^{i} y^{j}+O\left(|(x, y)|^{i+j+1}\right)\right\} .
$$

We say that $D_{0}$ satisfies the formal $N$-condition if this is a vector space for all $(i, j)$ with $2 \leq i+j \leq N$.

The semiformal condition. Let us denote, for $i \in \mathbb{N}, i \geq 1$,

$$
\begin{aligned}
J_{i}\left(D_{0}\right)= & \left\{A \mid A \text { is a germ of a map } \mathbb{R}^{c} \rightarrow \mathbb{R}^{c} \times \mathbb{R}\right. \\
& \text { and } \left.\exists H \in D_{0}: H(x, y)=(x, y)+A(x) y^{i}+O\left(y^{i+1}\right)\right\} .
\end{aligned}
$$

We say that $D_{0}$ satisfies the semiformal $N$-condition if $J_{i}\left(D_{0}\right)$ is a vector space for all $i \leq N$ and if the limit of a pointwise convergent sequence $A_{n}$ in $J_{i}\left(D_{0}\right)$, defined on some fixed neighbourhood of 0 , is again in $J_{i}\left(D_{0}\right)$

The path condition. Let $D_{1}$ be the set of germs of time $\tau$-dependent vector fields $Z_{\tau}, \tau \in[0,1]$, for which there exists a $C^{1}$ path of diffeomorphisms $\left(g_{\tau}\right)_{\tau \in[0,1]}$ in $D_{0}$ such that

or equivalently

$$
Z_{\tau}(v)=\frac{\partial g_{\tau}}{\partial \tau}\left(g_{\tau}^{-1}(v)\right)
$$

$$
Z_{\tau}\left(g_{\tau}(v)\right)=\frac{\partial g_{\tau}}{\partial \tau}(v)
$$

We say that $D_{0}$ satisfies the path $N$-condition if $D_{1}$ is a vector space and if the following three statements are true.

Statement 1. If $f \in D_{0}$ is of the form

$$
f(x, y)=N f(x, y)+A(x, y) y^{N}
$$

for some $A$ of class $C^{N}$, then there must exist a $C^{1}$ path $\left(A_{\tau}\right)_{\tau \in[0,1]}$ of $C^{N}$ maps such that $A_{0}=A, A_{1}=0$ and such that the diffeomorphism $f_{\tau}$ defined by $f_{\tau}(x, y):=$ $N f(x, y)+A_{\tau}(x, y) y^{N}$ is an element of $D_{0}$. So this means that the path $f_{\tau}$ connects $f$ with $N f$ within $D_{0}$.

Statement 2. For each element $\left(Z_{\tau}\right)_{\tau \in[0,1]}$ of $D_{1}$ the flow starting at the identity is in $D_{0}$, more precisely the solution $f_{\tau}$ of

must be in $D_{0}$.

$$
\left\{\begin{array}{l}
Z_{\tau}\left(f_{\tau}(v)\right)=\frac{\partial f_{\tau}}{\partial \tau}(v) \\
f_{0}=I d
\end{array}\right.
$$

Statement 3. The pointwise limit of a sequence in $D_{1}$, defined on some fixed neighbourhood of the origin, is again in $D_{1}$. 
Theorem 2. Let $N \in \mathbb{N}, N \geq 1$. Let $D_{0}$ be a group of germs in $0 \in \mathbb{R}^{c} \times \mathbb{R}$ of $C^{N}$ diffeomorphisms fixing the origin and satisfying the formal $2 N$, the semiformal $N$ and the path $N$-condition. Let $f$ be a $C^{2 N}$ element of $D_{0}$ satisfying the following properties:

(i) $d f(0)=\left[\begin{array}{cc}A_{c} & 0 \\ 0 & A_{s}\end{array}\right]$ where the eigenvalues of $A_{c}$ have modulus equal to 1 and $\left|A_{s}\right|<1$;

(ii) $\mathbb{R}^{c} \times\{0\}$ is a centermanifold for $f$;

(iii) $d f(0) \in D_{0}$.

Then there exists a $C^{N}$ element $H$ of $D_{0}$ such that

$$
H \circ f=N f \circ H .
$$

\section{(2.2) Proof of Theorem 2.}

(A) The formal part.

Proposition 1. There exists a $C^{2 N}$ element $H \in D_{0}$ such that

$$
j_{2 N}\left(H \circ f \circ H^{-1}\right)(0)=j_{2 N}(N f)(0) .
$$

$\left(j_{2 N}\right.$ denotes the Taylor polynomial of order $2 N$.)

Proof. This follows from the formal $2 N$ condition and from Broer's methods in [Br]. For completeness sake we sketch the big steps. The elimination of higher order terms goes inductively, and if $g=f-N f$ takes the form

$$
g(x, y)=A x^{i} y^{j}+\sum_{\substack{p+q=i+j \\(p, q) \neq(i, j)}} a_{p q} x^{p} y^{q}+O\left(|(x, y)|^{i+j+1}\right),
$$

then we can eliminate the term in $x^{i} y^{j}$ by a change of variables

$$
H(x, y)=(x, y)+C x^{i} y^{j}+O\left(|(x, y)|^{i+j+1}\right)
$$

not affecting the other terms of order $i+j$, as can be seen by equating and computing a bit, and where $C=(c, d)$ is a solution of

$$
(c, d)-\left(A_{c}^{-1} c \cdot A_{c}^{i} A_{s}^{j}, A_{s}^{-1} d \cdot A_{c}^{i} A_{s}^{j}\right)=\left(A_{c}^{-1} a, A_{s}^{-1} b\right) .
$$

The fact that this equation actually has a solution follows from the assumption about the eigenvalues of $d f(0)$ in Theorem 2 ; more concretely the linear map $(c, d) \mapsto$ $\left(A_{c} c, A_{s} d\right)-\left(c . A_{c}^{i} A_{s}^{j}, d . A_{c}^{i} A_{s}^{j}\right)$ is invertible. The proposition immediately follows from the next lemma:

Lemma 1. The solution $C=(c, d)$ of the foregoing equation is an element of $I_{i j}\left(D_{0}\right)$.

Proof. Let $C^{\prime}=\left(c^{\prime}, d^{\prime}\right) \in I_{i j}\left(D_{0}\right)$. By definition there exists $H^{\prime} \in D_{0}$ such that $H^{\prime}(x, y)=(x, y)+C^{\prime} x^{i} y^{j}+O\left(|(x, y)|^{i+j+1}\right)$. Put $H_{1}=d f(0)^{-1} \circ H^{\prime} \circ f$. Then $H_{1} \in D_{0}$. So $H_{1}(x, y)=(x, y)+\left(c_{1}, d_{1}\right) x^{i} y^{j}+O\left(|(x, y)|^{i+j+1}\right.$ with $C_{1}=\left(c_{1}, d_{1}\right) \in$ $I_{i j}\left(D_{0}\right)$. Equating terms of order $x^{i} y^{j}$ in the equality $d f(0) \circ H_{1}=H^{\prime} \circ f$ gives

$$
\left(c_{1}, d_{1}\right)-\left(A_{c}^{-1} a, A_{s}^{-1} b\right)=\left(A_{c}^{-1} c^{\prime} \cdot A_{c}^{i} A_{s}^{j}, A_{s}^{-1} d^{\prime} \cdot A_{c}^{i} A_{s}^{j}\right) .
$$


Expressing the fact that $d f(0)^{-1} \circ f \in D_{0}$ quickly yields $\left(A_{c}^{-1} a, A_{s}^{-1} b\right) \in I_{i j}\left(D_{0}\right)$. This last space is a vector space. From this we infer that the operator $T$ defined by $T .\left(c^{\prime}, d^{\prime}\right)=\left(A_{c}^{-1} c^{\prime} . A_{c}^{i} A_{s}^{j}, A_{s}^{-1} d^{\prime} . A_{c}^{i} A_{s}^{j}\right)$ maps $I_{i j}\left(D_{0}\right)$ into itself. From the solution of $(\times)$ we see that $I-T$ is invertible. Since we just obtained that $(I-T) I_{i j}\left(D_{0}\right) \subset$ $I_{i j}\left(D_{0}\right)$ we know from linear algebra that necessarily $I_{i j}\left(D_{0}\right)=(I-T)^{-1} I_{i j}\left(D_{0}\right)$. This proves the lemma and Proposition 1.

NOTE. The invertibility of the linear map just mentioned reflects the fact that there are some so called nonresonance conditions between the eigenvalues. More specifically let $\mu_{1}, \ldots, \mu_{c}$ be the eigenvalues of $A_{c}$. Then all terms, in the Taylor series of $f_{x}$, with at least one factor $y$ can be eliminated since for all $k \in\{1, \ldots, c\}$, all $j_{1}, \ldots, j_{c}$ and all $j \geq 1$

$$
\mu_{k} \neq \mu_{1}^{j_{1}} \ldots \mu_{c}^{j_{c}} A_{s}^{j}
$$

(take the modulus to see this). For a similar reason the terms, in the Taylor series of $f_{y}$, with at least a factor $y^{2}$ can be eliminated.

(B) The semiformal part.

From the 'formal part' (A) it follows that we may assume that $f$ takes the following form:

$$
f(x, y)=\left(f_{x}(x, 0)+y a(x, y), \frac{\partial f_{y}}{\partial y}(x, 0) \cdot y+y^{2} b(x, y)\right)
$$

where $a(x, y)=O\left(|(x, y)|^{2 N-1}\right)$ is of class $C^{2 N-1}$ and $y b(x, y)=O\left(|(x, y)|^{2 N-1}\right)$ is of class $C^{2 N-1}$.

We try to eliminate terms with 'low powers of $y$ '. This cannot be done on the formal level, and we have to use changes of variables $H$ of finite order of differentiability:

Proposition 2. There exist a $C^{N}$ germ of a diffeomorphism $H$ in $D_{0}$ (a change of variables) such that $\tilde{f}=H \circ f \circ H^{-1}$ is of the form

$$
\tilde{f}(x, y)=\left(f_{x}(x, 0)+y^{N} \tilde{a}(x, y), \frac{\partial f_{y}}{\partial y}(x, 0) \cdot y+y^{N} \tilde{b}(x, y)\right)
$$

with $(\tilde{a}, \tilde{b})(x, y)=O\left(|(x, y)|^{N}\right)$ of class $C^{N}$.

Proof. We proceed by induction on $i, 1 \leq i \leq N-1$, and assume that $f$ takes the form

$$
f(x, y)=\left(f_{x}(x, 0)+y^{i} a(x, y), \frac{\partial f_{y}}{\partial y}(x, 0) \cdot y+y^{i} b(x, y)\right)=:(u, v)
$$

where $(a, b)(x, y)=O\left(|(x, y)|^{2 N-i}\right)$ is of class $C^{2 N-i}$. The case $i=1$ is done. Let us abbreviate $M=2 N-i$. We can write $a(x, y)=a(x, 0)+O(y)$. We try to find a change of variables $H$ of the form

$$
H(x, y)=\left(x+c(x) y^{i}, y+d(x) y^{i}\right)+O\left(y^{i+1}\right)=:\left(x^{\prime}, y^{\prime}\right)
$$

such that $(c, d)(x)=O\left(|x|^{M}\right)$ is of class $C^{M}$ as well as $H$. We want to have that $H \circ f=\tilde{f} \circ H$ where

$$
\tilde{f}(x, y)=\left(f_{x}(x, 0), \frac{\partial f_{y}}{\partial y}(x, 0) \cdot y\right)+O\left(y^{i+1}\right) .
$$


This gives an equation of the form

$$
\left\{\begin{array}{l}
u+c(u) v^{i}=f_{x}\left(x^{\prime}, 0\right)+O\left(y^{i+1}\right) \\
v+d(u) v^{i}=\frac{\partial f_{y}}{\partial y}\left(x^{\prime}, 0\right) \cdot y^{\prime}+O\left(y^{i+1}\right)
\end{array}\right.
$$

so we have to solve in $(c, d)$ (a few calculations):

$$
\left\{\begin{aligned}
a(x, 0)+c\left(f_{x}(x, 0)\right) \cdot \frac{\partial f_{y}}{\partial y}(x, 0)^{i} & =\frac{\partial f_{x}}{\partial x}(x, 0) c(x) \\
b(x, 0)+d\left(f_{x}(x, 0)\right) \frac{\partial f_{y}}{\partial y}(x, 0)^{i} & =\frac{\partial f_{y}}{\partial y}(x, 0) \cdot d(x) .
\end{aligned}\right.
$$

Let us denote

$$
\bar{A}(x)=(\bar{a}, \bar{b})(x):=\left(\frac{\partial f_{x}}{\partial x}(x, 0)^{-1} \cdot a(x), \frac{\partial f_{y}}{\partial y}(x, 0)^{-1} \cdot b(x)\right)
$$

and let $T$ be the operator acting on germs of functions $A=(a, b): \mathbb{R}^{c} \rightarrow \mathbb{R}^{c} \times \mathbb{R}$ defined by

$$
T A(x)=\left(\frac{\partial f_{x}}{\partial x}(x, 0)^{-1} \cdot a\left(f_{x}(x, 0)\right) \cdot \frac{\partial f_{y}}{\partial y}(x, 0)^{i}, \frac{\partial f_{y}}{\partial y}(x, 0)^{-1} b\left(f_{x}(x, 0)\right) \frac{\partial f_{y}}{\partial y}(x, 0)^{i}\right) .
$$

Then the foregoing becomes

$$
\bar{A}(x)+T(c, d)(x)=(c, d) .
$$

In the sequel we will assume that the derivative of $f$ satisfies globally certain inequalities, based on the assumption (i) about the eigenvalues of $d f(0)$ in Theorem 2. We can obtain them by using 'cut off' functions, i.e. functions being 1 on a neighbourhood of the origin and being 0 outside some bigger neighbourhood. This construction is described in detail in [Bo] and will not be repeated here for shortness sake.

Lemma 2. There exists $\beta<1$ and a neighbourhood $U$ of $x=0$ in $\mathbb{R}^{c}$ such that, assuming a decent 'cut off' construction for $f$, the following statement holds.

Let $E>0$. If $A$ is a $C^{M} \operatorname{map} U \rightarrow \mathbb{R}^{c} \times \mathbb{R}$ satisfying $|A(x)| \leq E|x|^{M}, \ldots$, $\left|d^{j} A(x)\right| \leq E|x|^{M-j}, \ldots,\left|d^{M} A(x)\right| \leq E$ for all $x \in U$ then $T A$ is of class $C^{M}$ and $|T A(x)| \leq \beta E|x|^{M}, \ldots,\left|d^{j} T A(x)\right| \leq \beta E|x|^{M-j}, \ldots,\left|d^{M} T A(x)\right| \leq \beta E$ for all $x \in U$.

Proof. Let us write $A=(a, b)$ with respect to the product $\mathbb{R}^{c} \times \mathbb{R}$ and let us treat the components of $T A$ separately. In terms of the abbreviations

$$
L(x)=\frac{\partial f_{x}}{\partial x}(x, 0)^{-1}, \quad B(x)=\frac{\partial f_{y}}{\partial y}(x, 0)^{i}, \quad \bar{f}(x)=f_{x}(x, 0)
$$

we can write the $\mathbb{R}^{c}$ component of $T A(x)$ as

$$
T_{1} a(x):=L(x) \cdot a(\bar{f}(x)) \cdot B(x) .
$$


Let $1 \leq j \leq M$. From a straightforward use of Leibnitz' rule for differentiation of a product (or in general: of a bilinear operation '.') and from the higher order chain rule we can derive an expression of the form

$$
\left|d^{j}\left(T_{1} a\right)(x)\right| \leq\left|L(x) \cdot d^{j} a(\bar{f}(x)) \cdot(d \bar{f}(x))^{j} \cdot B(x)\right|+C(f, j) \sum_{k=0}^{j-1}\left|d^{k} a(\bar{f}(x))\right|
$$

where $C(f, j)$ is some constant depending in a polynomial way on the bounds of the derivatives of order 1 to $j$ of $f$ (remember: a decent 'cut off' is assumed here). One can be more explicit about this constant, but we don't need this here. The first term in the right hand side can be estimated as follows $\left(|\cdot|_{\infty}\right.$ denotes the supremum norm)

$$
\left|L(x) \cdot d^{j} a(\bar{f}(x)) \cdot(d \bar{f}(x))^{j} \cdot B(x)\right| \leq|L|_{\infty} E\left(|d \bar{f}|_{\infty}|x|\right)^{M-j}|d \bar{f}|_{\infty}^{j}|B|_{\infty} .
$$

Because of assumption (i) in Theorem 2 about the eigenvalues we can arrange that

$$
\alpha:=|L|_{\infty}\left(|d \bar{f}|_{\infty}\right)^{M}|B|_{\infty}<1
$$

so

$$
\left|L(x) \cdot d^{j} a(\bar{f}(x)) \cdot(d \bar{f}(x))^{j} \cdot B(x)\right| \leq \alpha E|x|^{M-j} .
$$

The remaining terms can be estimated by

$$
C(f, j) E\left(|d \bar{f}|_{\infty}|x|\right)^{M-j+1} .
$$

We can summarize this by

$$
\left|d^{j}\left(T_{1} a\right)(x)\right| \leq E|x|^{M-j}\left[\alpha+C^{\prime}(f, j)|x|\right]
$$

where $C^{\prime}$ is some other constant.

The second $(\mathbb{R}-)$ component of $T A(x)$ is estimated in a completely similar way; this time the key inequality is

$$
\left|\frac{\partial f_{y}}{\partial y}(x, 0)^{-1}\right| .\left|\frac{\partial f_{y}}{\partial y}(x, 0)^{i}\right|<1
$$

we obtain an $\alpha^{\prime}<1$ and an estimate of the form

$$
\left|d^{j}(T A)(x)\right| \leq E|x|^{M-j}\left[\alpha^{\prime}+C^{\prime \prime}(f, j)|x|\right] .
$$

If we choose $\alpha^{\prime}<\beta<1$, Lemma 2 quickly follows from this.

We proceed with the proof of Proposition 2. From Lemma 2 it follows that the series $\sum_{s=0}^{\infty} T^{s} \bar{A}$ as well as its derivatives up to order $M$ converge uniformly on $U$, since they are dominated by the geometric series $E \sum_{s=0}^{\infty} \beta^{s}$. We conclude that $(c, d):=\sum_{s=0}^{\infty} T^{s} \bar{A}$ is a $C^{M}$ solution of (2), satisfying moreover $\left|d^{j}(c, d)(x)\right| \leq$ $\frac{1}{1-\beta} E|x|^{M-j}$. 
Lemma 3. (i) $\bar{A} \in J_{i}\left(D_{0}\right)$ and

(ii) $A \in J_{i}\left(D_{0}\right) \Rightarrow T A \in J_{i}\left(D_{0}\right)$.

Proof. (i) Consider $H_{1}:=N f^{-1} \circ f$. By the path condition surely $N f \in D_{0}$ and hence $H_{1} \in D_{0}$. By the definition of $J_{i}\left(D_{0}\right)$ the coefficient of $y^{i}$ in the development of $H_{1}$ is an element of it. More concretely let us write $H_{1}(x, y)=$ $(x, y)+\left(a_{1}(x), b_{1}(x)\right) y^{i}+O\left(y^{i+1}\right)$. Then $\left(a_{1}, b_{1}\right) \in J_{i}\left(D_{0}\right)$. On the other hand we express the fact that $N f \circ H_{1}=f$. After calculating a bit we can equate terms in $y^{i}$ and obtain that $\left(a_{1}, b_{1}\right)$ is in fact equal to $\bar{A}$, so $\bar{A} \in J_{i}\left(D_{0}\right)$.

(ii) Let $A \in J_{i}\left(D_{0}\right)$ and let $H \in D_{0}$ be such that $H(x, y)=(x, y)+A(x) y^{i}+$ $O\left(y^{i+1}\right)$. Like in (i) we have that $H_{1}:=N f^{-1} \circ H \circ f \in D_{0}, H_{1}(x, y)=(x, y)+$ $\left(c_{1}(x), d_{1}(x)\right) y^{i}+O\left(y^{i+1}\right)$ with $\left(c_{1}, d_{1}\right) \in J_{i}\left(D_{0}\right)$. Again, equating terms in $y^{i}$ in the equation $N f \circ H_{1}=H \circ f$ and calculating gives $\left(c_{1}, d_{1}\right)=T A+\bar{A}$. Using part (i) we infer that $T A \in J_{i}\left(D_{0}\right)$.

Now we can finish the proof of Proposition 2. We have $\bar{A} \in J_{i}\left(D_{0}\right)$. By induction $T^{s} \bar{A} \in J_{i}\left(D_{0}\right), \forall s \in \mathbb{N}$. Since $J_{i}\left(D_{0}\right)$ is a vector space every partial sum $\sum_{s=0}^{n} T^{s} \bar{A}$ is in $J_{i}\left(D_{0}\right)$. Finally the condition about pointwise convergence implies that $(c, d) \in$ $J_{N}\left(D_{0}\right)$, so that there exists $H \in D_{0}$ of the form

$$
H(x, y)=(x, y)+(c, d)(x) y^{i}+O\left(y^{i+1}\right)
$$

such that $H \circ f=\tilde{f} \circ H$.

(C) The 'path' part.

We continue with the proof of Theorem 2. Because $N f$ is not necessarily linear (giving a nonlinear equation for $H$ ) we want to use the 'linearization' method of the path. We describe it briefly, and refer to [DRR, R, M] for more details.

The ideas of the sequel are similar to those in [Bo]. For the sake of being self contained we copy some of them briefly and give the main steps in the estimates. All the occurring objects can be defined globally on $\mathbb{R}^{c} \times \mathbb{R}$ by using cut off functions: for details we refer to [Bo].

Because of Proposition 2 we may assume that $f$ has the form

$$
f(x, y)=\left(f_{x}(x, 0)+y^{N} a(x, y), \frac{\partial f_{y}}{\partial y}(x, 0) \cdot y+y^{N} b(x, y)\right)
$$

with $A(x, y):=(a, b)(x, y)=O\left(|(x, y)|^{N}\right)$ of class $C^{N}$. By the path $N$-condition there exists a $C^{1}$ path $\left(A_{\tau}\right)_{\tau \in[0,1]}$ connecting $A$ with 0 such that

$$
f_{\tau}(x, y):=N f(x, y)+A_{\tau}(x, y) y^{N}
$$

is a path in $D_{0}$; this last path connects $f$ with $N f$. We look for a $C^{1}$ path of diffeomorphisms $\Psi_{\tau}$ in $D_{0}$ such that

$$
\left\{\begin{array}{l}
\Psi_{\tau} \circ f=f_{\tau} \circ \Psi_{\tau} \\
\Psi_{0}=\text { Identity }
\end{array}\right.
$$

The variables are abbreviated by $(x, y)=: v$ and $(\tau, v)=: w$; we also denote $|w|_{\Sigma}=$ $|y|$, this is the distance to $\Sigma:=[0,1] \times \mathbb{R}^{c} \times\{0\}$. 
Putting $G(\tau, v)=f_{\tau}(v)$ and $\Omega(\tau, v)=\Psi_{\tau}(v)$ this means that we have to solve (in $\Omega$ ):

$$
\left\{\begin{array}{l}
G\left(\tau, \Omega\left(\tau, f^{-1}(v)\right)\right)=\Omega(\tau, v) \\
\Omega(0, .)=\text { Identity. }
\end{array}\right.
$$

We differentiate the first equation with respect to $\tau$, then we replace $v$ by $\Psi_{\tau}^{-1}(v)$. After a calculation this gives, using $\Psi_{\tau} \circ f^{-1} \circ \Psi_{\tau}^{-1}=f_{\tau}^{-1}$ and denoting

$$
\begin{aligned}
& Z(\tau, v)=\frac{\partial \Omega}{\partial \tau}\left(\tau, \Psi_{\tau}^{-1}(v)\right) \text { and } \\
& X(\tau, v)=\left(d f_{\tau}(v)\right)^{-1} \cdot \frac{\partial G}{\partial \tau}(\tau, v),
\end{aligned}
$$

the following linear equation in $Z$ :

$$
Z(\tau, v)-d f_{\tau}^{-1}\left(f_{\tau}(v)\right) \cdot Z\left(\tau, f_{\tau}(v)\right)=X(\tau, v) .
$$

Lemma 4. One has

$$
X(\tau, v)=-\frac{\partial f_{\tau}^{-1}}{\partial \tau}\left(f_{\tau}(v)\right)
$$

Proof. Write $h_{\tau}=f_{\tau}^{-1}, G(\tau,)=.f_{\tau}$ and $H(\tau,)=.h_{\tau}$. First

$$
h_{\tau} \circ f_{\tau}=I d
$$

so

$$
d h_{\tau}\left(f_{\tau}(v)\right) \circ d f_{\tau}(v)=I d
$$

Second

$$
H(\tau, G(\tau, v))=v
$$

so differentiating with respect to $\tau$ gives

$$
\frac{\partial H}{\partial \tau}(\tau, G(\tau, v))+\partial_{2} H(\tau, G(\tau, v)) \cdot \frac{\partial f_{\tau}}{\partial \tau}(v)=0
$$

and since

$$
\partial_{2} H(\tau, G(\tau, x))=d h_{\tau}\left(f_{\tau}(v)\right)=d f_{\tau}(v)^{-1}
$$

we find

$$
\begin{aligned}
\left(d f_{\tau}(v)\right)^{-1} \cdot \frac{\partial f_{\tau}}{\partial \tau}(v) & =\partial_{2} H(\tau, G(\tau, x)) \cdot \frac{\partial f_{\tau}}{\partial \tau}(v) \\
& =-\frac{\partial H}{\partial \tau}(\tau, G(\tau, v))=-\frac{\partial f_{\tau}^{-1}}{\partial \tau}\left(f_{\tau}(v)\right) .
\end{aligned}
$$

We consider $Z_{\tau}=Z(\tau,$.$) and X_{\tau}=X(\tau,$.$) as time \tau$-dependent vector fields. By Lemma 4 we see that $X_{\tau} \in D_{1}$ (cf. the path condition). We try to find a solution $Z$ of (5) such that $Z_{\tau}$ is also in $D_{1}$. Because then the solution $\Psi_{\tau}=\Omega(\tau,$.$) of the$ initial value problem

$$
\left\{\begin{array}{l}
\frac{\partial \Omega}{\partial \tau}(\tau, v)=Z(\tau, \Omega(\tau, v)) \\
\Omega(0, .)=\text { Identity }
\end{array}\right.
$$

is a solution for (3), as was checked in $[\mathrm{M}]$, so $\Psi_{1}$ will solve our original problem and will be in $D_{0}$. 
Solution of equation (5). The idea is similar to the one in the proof of Proposition 2. This time we consider the operator $L$ defined by

$$
(L X)_{\tau}(v):=d f_{\tau}^{-1}\left(f_{\tau}(v)\right) \cdot X_{\tau}\left(f_{\tau}(v)\right) .
$$

Let us put

$$
\mathcal{X}:=\left\{X:[0,1] \times \mathbb{R}^{c} \times \mathbb{R} \rightarrow \mathbb{R}^{c} \times \mathbb{R}: X(\tau, x, 0) \equiv 0\right\}
$$

and for $N \in \mathbb{N}$ and $E>0$ :

$$
\begin{aligned}
& \mathcal{X}(N, E)=\left\{X: X \in \mathcal{X} \text { and } X \text { is of class } C^{N}\right. \text { and } \\
& X(\tau, x, 0)=\frac{\partial X}{\partial y}(\tau, x, 0)=\cdots=\frac{\partial^{N} X}{\partial y^{N}}(\tau, x, 0)=0 \\
&\text { and } \left.\left|\frac{\partial^{N} X}{\partial y^{N}}(\tau, x, y)\right| \leq E\right\} .
\end{aligned}
$$

Observe that $X$ obtained by the method of the path is an element of $\mathcal{X}\left(N,\left|d^{N} X\right|_{\infty}\right)$, always assuming a decent cut off for $f$.

Lemma 5. There exists $\beta<1$ such that for all $E>0$, all $X \in \mathcal{X}(N, E)$ and all $j \in \mathbb{N}$ the following estimate holds:

$$
\left|L^{j} X(w)\right| \leq \beta^{j} E|w|_{\Sigma}^{N}
$$

for all $w \in[0,1] \times \mathbb{R}^{c} \times \mathbb{R}$.

Proof. As

$$
\left|L^{j} X(\tau, v)\right| \leq\left|d f_{\tau}^{-1}\right|_{\infty}^{j}\left|X\left(\tau, f_{\tau}^{j}(v)\right)\right|
$$

and as, by the invariance of $\mathbb{R}^{c} \times\{0\}$ for $f_{\tau}$,

$$
\left|\pi_{y} \circ f_{\tau}(x, y)\right| \leq\left|\frac{\partial\left(\pi_{y} \circ f_{\tau}\right)}{\partial y}\right|_{\infty}|y|
$$

( $\pi_{y}$ is the projection on the $\mathbb{R}$ component) we get

$$
\left|L^{j} X(\tau, v)\right| \leq\left[\left|d f_{\tau}^{-1}\right|_{\infty} \cdot\left|\frac{\partial\left(\pi_{y} \circ f_{\tau}\right)}{\partial y}\right|_{\infty}^{N}\right]^{j} E|(\tau, v)|_{\Sigma}^{N}
$$

It is not difficult to see that Lemma 5 now follows from assumption (i) about the eigenvalues in Theorem 2 if we use cut off functions (cf. [Bo]).

Consequently, for each $X \in \mathcal{X}(N, E)$ the series $\sum_{j=0}^{\infty} L^{j} X$ converges locally uniformly to a continuous solution $Z$ of (5).

Now we show that if $X_{\tau}$ is in $D_{1}$ then also $(L X)_{\tau}$ is. Let

$$
X_{\tau}(v)=\frac{\partial g_{\tau}}{\partial \tau}\left(g_{\tau}^{-1}(v)\right)
$$


with $g_{\tau} \in D_{0}$. Put $h_{\tau}=f_{\tau}^{-1} \circ g_{\tau}$. Then

$$
\frac{\partial h_{\tau}}{\partial \tau}(u)=\frac{\partial f_{\tau}^{-1}}{\partial \tau}\left(g_{\tau}(u)\right)+d f_{\tau}^{-1}\left(g_{\tau}(u)\right) \cdot \frac{\partial g_{\tau}}{\partial \tau}(u)
$$

if we replace

$$
u=h_{\tau}^{-1}(v)=g_{\tau}^{-1} \circ f_{\tau}(v)
$$

then we obtain

$$
\begin{aligned}
\frac{\partial h_{\tau}}{\partial \tau}\left(h_{\tau}^{-1}(v)\right) & =\frac{\partial f_{\tau}^{-1}}{\partial \tau}\left(f_{\tau}(v)\right)+d f_{\tau}^{-1}\left(f_{\tau}(v)\right) \cdot \frac{\partial g_{\tau}}{\partial \tau}\left(g_{\tau}^{-1} \circ f_{\tau}(v)\right) \\
& =\frac{\partial f_{\tau}^{-1}}{\partial \tau}\left(f_{\tau}(v)\right)+d f_{\tau}^{-1}\left(f_{\tau}(v)\right) \cdot X_{\tau}\left(f_{\tau}(v)\right) \\
& =\frac{\partial f_{\tau}^{-1}}{\partial \tau}\left(f_{\tau}(v)\right)+(L X)_{\tau}(v) .
\end{aligned}
$$

As $h_{\tau}$ and $f_{\tau}^{-1}$ are both paths in $D_{0}$ and as $D_{1}$ is a vector space, we see that the path $(L X)_{\tau}$ is again in $D_{1}$.

By induction every partial sum of the series $\sum_{j>0} L^{j} X$ is in $D_{1}$, and by the third statement in the path condition we infer that our solution $Z$ of (5) is in $D_{1}$, as desired.

Let us finally show that $Z$ is of class $C^{N}$.

Lemma 6. There exist $\beta<1$ and $\varepsilon>0$ with the following property. Let $E>0$. If $X \in \mathcal{X}$ is of class $C^{N}$ and if for all $|w|_{\Sigma} \leq \varepsilon$ and $0 \leq i \leq N$

$$
\left|d^{i} X(w)\right| \leq E|w|_{\Sigma}^{N-i}
$$

then for all $|w|_{\Sigma} \leq \varepsilon$ and $0 \leq i \leq N$

$$
\left|d^{i}(L X)(w)\right| \leq \beta E|w|_{\Sigma}^{N-i}
$$

Proof. Let us put shortly $w^{\prime}=\left(\tau, v^{\prime}\right)=\left(\tau, f_{\tau}(v)\right)$. Just like in Lemma 2 we can make an estimate

$$
\left|d^{i}(L X)(w)\right| \leq\left|d f_{\tau}^{-1}\left(v^{\prime}\right) \cdot d^{i} X\left(w^{\prime}\right) \cdot\left(d f_{\tau}(v)\right)^{i}\right|+C(f, i) \sum_{k=0}^{i-1}\left|d^{k} X\left(w^{\prime}\right)\right|
$$

where $C(f, i)$ is some constant depending in a polynomial way on the bounds of the derivatives of order 1 to $i+1$ of $f$. The first term in the latter right hand side is estimated by

$$
\left|d f_{\tau}^{-1}\left(v^{\prime}\right) \cdot d^{i} X\left(w^{\prime}\right) \cdot\left(d f_{\tau}(v)\right)^{i}\right| \leq\left|d f_{\tau}^{-1}\right| E\left|\frac{\partial\left(\pi_{y} \circ f_{\tau}\right)}{\partial y}\right|_{\infty}^{N-i}|w|_{\Sigma}^{N-i}\left|d f_{\tau}\right|_{\infty}^{i}
$$

and for the second term in it we observe that

$$
\left|d^{k} X\left(w^{\prime}\right)\right| \leq E\left|w^{\prime}\right|_{\Sigma}^{N-k} \leq E|w|_{\Sigma}^{N-k}
$$


so we conclude that

$$
\left|d^{i}(L X)(w)\right| \leq E|w|_{\Sigma}^{N-i}\left[\left|d f_{\tau}^{-1}\right|\left|\frac{\partial\left(\pi_{y} \circ f_{\tau}\right)}{\partial y}\right|_{\infty}^{N-i}\left|d f_{\tau}\right|_{\infty}^{i}+i C(f, i)|w|_{\Sigma}\right] .
$$

The lemma now follows from assumption (i) about the eigenvalues in Theorem $2 . \square$

From this lemma we derive that the $i$-th derivative of the series $\sum_{j=0}^{\infty} L^{j} X$ converges uniformly on the strip $|w|_{\Sigma} \leq \varepsilon$, since it is dominated by the geometric series $\sum_{j=0}^{\infty} \beta^{j} E|w|_{\Sigma}^{N-i}$. Consequently the limit $Z$ is of class $C^{N}$ on that strip; because of the dynamical behavior in the $\mathbb{R}$ direction it is $C^{N}$ everywhere.

Let us finally come to the solution of (6). From the foregoing lemma it follows that $|Z(w)| \leq \frac{1}{1-\beta} E|w|_{\Sigma}^{N}$ on some strip $|w|_{\Sigma} \leq \varepsilon$. Consider the differential equation $\frac{d u}{d t}=\frac{1}{1-\beta} E u^{N}, u \in \mathbb{R}^{+}$. The solution of this with initial value $u(0)=u_{0}$ will not blow up for $t \in[0,1]$ provided that $u_{0}<\left(\frac{E(N-1)}{1-\beta}\right)^{\frac{1}{-N+1}}$. Consequently the solution $\Psi_{1}=\Omega(1,$.$) will exist on the region |w|_{\Sigma}<\left(\frac{E(N-1)}{1-\beta}\right)^{\frac{1}{-N+1}}$.

This finishes the proof of Theorem 2 .

\section{EXAMPLES}

Now we show that the motivating example (Theorem 1) of section 1 satisfies the formal, semiformal and path conditions for any $N \geq 1$. So write, for the centermanifold, $\mathbb{R}^{c}=\mathbb{R}^{P} \times \mathbb{R}^{2}$ and $x=\left(\lambda, x_{1}, x_{2}\right) \in \mathbb{R}^{P} \times \mathbb{R}^{2} . \lambda$ is to be interpreted as a parameter, and $\left(x_{1}, x_{2}\right)$ are the 'actual' center variables.

Let $p, q \in \mathbb{N}$ be given, $p, q \geq 1$. Let $D_{0}$ consist of those germs of diffeomorphisms $f$ of $\mathbb{R}^{c} \times \mathbb{R}$ which leave the level sets of the function

$$
F\left(\lambda, x_{1}, x_{2}, y\right)=\left(\lambda, x_{1}^{p} x_{2}^{q}\right)
$$

invariant. Let us also write $f$ in components as $f=\left(f_{x}, f_{y}\right)=\left(f_{\lambda}, f_{1}, f_{2}, f_{y}\right) \in$ $\mathbb{R}^{P} \times \mathbb{R}^{2} \times \mathbb{R}$. Invariance means that $F\left(f\left(\lambda, x_{1}, x_{2}, y\right)\right)=F\left(\lambda, x_{1}, x_{2}, y\right)$. For the first component of $F$ this gives $f_{\lambda}\left(\lambda, x_{1}, x_{2}, y\right)=\lambda$. This reflects the fact that $\lambda$ is a parameter.

Necessarily the $x_{k}=0$ hyperplanes, $k=1,2$, are invariant. Hence we can write

$$
f_{k}\left(\lambda, x_{1}, x_{2}, y\right)=g_{k}\left(\lambda, x_{1}, x_{2}, y\right) \cdot x_{k}
$$

for some $g_{k}$. Invariance of the level sets of $F$ then implies that

$$
g_{1}^{p} g_{2}^{q}=1 \text {. }
$$

Let us now check the formal, semiformal and path conditions for $D_{0}$ for any $N \in \mathbb{N}$.

(A) The formal condition. We describe the elements of $I_{i j}\left(D_{0}\right)$. If $H \in D_{0}$ with $H=\left(\lambda, H_{1}, H_{2}, H_{y}\right)$ then we write, for the Taylor expansion, $H_{k}=$ $\left(1+h_{k}+\ldots\right) x_{k}, k=1,2$, and one must have

$$
\left(1+h_{1}+\ldots\right)^{p}=\left(1+h_{2}+\ldots\right)^{-q}
$$

(dots mean irrelevant higher order terms) so $p h_{1}=-q h_{2}$. We see that for an element $C=(c, d) \in I_{i j}\left(D_{0}\right)$ the term $c x^{i} y^{j}$ always is a multiple of $\left(0, q x_{1},-p x_{2}\right)$ (the 0-entry in the first component comes from the parameter $\lambda \in \mathbb{R}^{P}$; this will remain so in the sequel). Hence $I_{i j}\left(D_{0}\right)$ is a vector space. 
(B) The semiformal condition. Let us describe the elements of $J_{i}\left(D_{0}\right)$. Let $H=$ $\left(\lambda, H_{1}, H_{2}, H_{y}\right) \in D_{0}$. We consider its development in powers of $y$ and write $H_{k}=$ $\left(1+a_{k}(x) y^{N}+\ldots\right) x_{k}$. In the same way as in (A) the invariance of the level sets for $H$ implies that $p a_{1}=-q a_{2}$ and for an element $A=(a, b) \in J_{i}\left(D_{0}\right)$ its $a$-component must be a multiple of $\left(0, q x_{1},-p x_{2}\right)$. Conversely if $a(x)=\bar{a}(x)\left(0, q x_{1},-p x_{2}\right)$ for some function $\bar{a}$ the map

$$
H(x, y)=\left(\lambda,\left(1+q \bar{a}(x) y^{N}\right) \cdot x_{1},(1+q \bar{a}(x))^{-p / q} x_{2}, H_{y}(x, y)\right)
$$

is an element of $D_{0}$. We conclude that $A=(a, b) \in J_{i}\left(D_{0}\right)$ if and only if $a(x)$ is a multiple of $\left(0, q x_{1},-p x_{2}\right)$. Hence $J_{i}\left(D_{0}\right)$ is a vector space and the limit of a pointwise convergent sequence in it, defined on some fixed neighbourhood of 0 , is again in it.

(C) The path condition. As before we can write, for $x=\left(\lambda, x_{1}, x_{2}\right), f(x, y)=$ $\left(\lambda, g_{1}(x, y) \cdot x_{1}, g_{2}(x, y) \cdot x_{2}, f_{y}(x, y)\right)$ with $g_{1}^{p} g_{2}^{q}=1$. Hence

$$
N f(x, y)=\left(\lambda, g_{1}(x, 0) \cdot x_{1}, g_{2}(x, 0) \cdot x_{2}, \frac{\partial f_{y}}{\partial y}(x, 0) . y\right)
$$

Let us come to Statement 1 in the path condition. So write $f(x, y)=N f(x, y)+$ $A(x, y) y^{N}$, where $A(x, y)=\left(0, a_{1}(x, y) \cdot x_{1}, a_{2}(x, y) \cdot x_{2}, b(x, y)\right) \in \mathbb{R}^{p} \times \mathbb{R}^{3}$. A direct calculation shows that the path

$$
\begin{aligned}
f_{\tau}(x, y) & =\left(\lambda, g_{1}(x, 0)\left(1+\tau g_{1}(x, 0)^{-1} a_{1}(x, y) y^{N}\right) \cdot x_{1}\right. \\
& \left.g_{2}(x, 0) \cdot\left(1+\tau g_{1}(x, 0)^{-1} a_{1}(x, y) y^{N}\right)^{-p / q} \cdot x_{2}, \frac{\partial f_{y}}{\partial y}(x, 0) \cdot y+\tau b(x, y) y^{N}\right)
\end{aligned}
$$

connects $f$ with $N f$ within $D_{0}$ (i.e. one verifies that $f_{\tau}$ leaves the level sets of $F$ invariant). The coefficient of $y^{N}$ is the wanted $A_{\tau}$ in Statement 1.

Secondly, a time-dependent vector field $Z_{\tau}$ in $D_{1}$ is tangent to the level sets of $F$, so its flow will leave the level sets of $F$ invariant. This is Statement 2. Finally, Statement 3 of the path condition is trivial since a pointwise limit of such vector fields remains tangent to the level sets of $F$.

\section{Generalisations}

Let us indicate how to derive, in a standard way [DR1], the similar result for vector fields thanks to the uniqueness of the solutions of the equations in the proof of Theorem 2. Suppose that $X$ is a vector field near $0 \in \mathbb{R}^{c} \times \mathbb{R}$ such that its flow $X_{t}$ is in $D_{0}$. Suppose that $d X(0)=\left[\begin{array}{cc}A_{c} & 0 \\ 0 & A_{s}\end{array}\right]$ where

(i) the eigenvalues of $A_{c}$ are on the imaginary axis and those of $A_{s}$ have real part $<0$,

(ii) $\mathbb{R}^{c} \times\{0\}$ is a centermanifold for $X$;

(iii) $d\left(X_{t}\right)(0) \in D_{0}$ for every $t \in[0,1]$.

Let us in fact show that the diffeomorphism $H \in D_{0}$, obtained from the Main Theorem 2, conjugating the time one of $X$ to the time one of $N X$, actually conjugates the flow for all times $t \in[0,1]$. We claim in fact that $\Psi_{t}:=X_{-t} \circ H^{-1} \circ N X_{t} \circ H$ equals the identity. A straightforward calculation [DR1] shows that $N X_{1} \circ \Psi_{t}=$ 
$\Psi_{t} \circ N X_{1}$ (index 1 means time one). Now we are in the situation of the 'path part' (C) of the proof of Theorem 2, this time with $f=f_{t}=N X_{1}$. We obtain an equation

$$
Z(t, v)-d f^{-1}(f(v)) \cdot Z(t, f(v))=0
$$

with $Z(t, v)=\frac{\partial \Psi_{t}}{\partial t}\left(t, \Psi_{t}^{-1}(v)\right)$. From the estimates in Lemma 5 it follows that this equation has a unique solution, thanks to the fact that $d f$ satisfies certain global estimates. We conclude that $Z=0$ and $\Psi_{t}$ is the identity.

Question. Suppose that we would like to replace the group of diffeomorphisms $D_{0}$ in the result by some Lie subalgebra $L_{0}$ of vector fields. One could ask that the change of variables $H$ conjugating $X$ to $N X$ be the time $t$ of some element of $L_{0}$. The methods we use do not imply this result, e.g. the change of variables $\Psi_{1}$ obtained by the method of the path in part $(\mathrm{C})$ of the proof is the time one of a time-dependent vector field.

Higher codimensions. In case that the codimension of the centermanifold is more than one, some nonresonance conditions on the eigenvalues of $d f(0)$ have to be satisfied in order to have a sufficiently differentiable change of coordinates. Suppose that $f$ satisfies the Sternberg $\alpha(d f(0), k)$-condition as defined by Takens in [T]. Then we expect that one can obtain a $C^{k}$ element $H$ of $D_{0}$ if $D_{0}$ satisfies the formal, semiformal and path conditions for $N$ sufficiently large with respect to $k$. We believe that the proof may follow the same lines but part (B) of it (the semiformal part) will however be considerably more technical, cf. Takens' original proof in $[\mathrm{T}]$. Also part $(\mathrm{C})$ will have to be split up in stable and unstable directions.

The methods we developed here can be applied to other types of normal form theorems. For example we can prove in a very comparable way the following result about hyperbolic singularities in the plane, suggested by F. Dumortier:

Theorem 3. Let $X$ be a $C^{\infty}$ vector field near $0 \in \mathbb{R}^{2}, d X(0)$ hyperbolic. Suppose that $X . F=0$ where $F(x, y)=x^{p} y^{q}$ with $(p, q)=1$. Then there exists a $C^{\infty}$ change of variables $\varphi$ near 0 satisfying

(i) $\varphi_{*} X(x, y)=g\left(x^{p} y^{q}\right)\left[q x \frac{\partial}{\partial x}-p y \frac{\partial}{\partial y}\right]$ and

(ii) $F(\varphi(x, y))=F(x, y)$ i.e. $\varphi$ respects the level sets of $F$.

\section{REFERENCES}

[BP] Beloqui, J. and Pacifico, M.J., Quasi-transversal saddle-node bifurcation on surfaces, Ergod. Th. \& Dynam. Sys. 10 (1990), 63-88. MR 92a:58104

[Bo] Bonckaert, P., On the continuous dependence of the smooth change of coordinates in parametrized normal form theorems, Journal of Differential Equations 106 (1993), 107120. MR 94j:58150

[Br] Broer, H., Formal normal form theorems for vector fields and some consequences in the volume preserving case, Dynamical systems and Turbulence, Warwick, Lecture Notes in Math. 898, Springer-Verlag, Berlin-Heidelberg-New York, 1981. MR 83j:58085

[CP] Carneiro, M. and Palis, J., Bifurcations and global stability of families of gradients, Publ. Math. IHES 70 (1989), 103-168. MR 91f:58048

[DRR] Dumortier, F. D., Rodrigues, P. and Roussarie, R., Germs of diffeomorphisms in the plane, Lecture Notes in Mathematics 902, Springer-Verlag, 1981. MR 83f:58008

[DR1] Dumortier, F. and Roussarie. R., Germes de difféomorphismes et de champs de vecteurs en classe de différentiabilité finie, Ann. Inst. Fourier 33 (1983), 195-267. MR 84m:58114 
[DR2] Dumortier, F. and Roussarie, R., Canard cycles and center manifolds, Memoirs of the AMS (to appear).

[IY] $\mathrm{Il}^{\prime}$ yashenko, Y. and Yakovenko, S., Finitely smooth normal forms of local families of diffeomorphisms and vector fields, Russian Math. Surveys 46:1 (1991), 1-43. MR 92i:58165

[M] Mather, J., Stability of $C^{\infty}$ mappings: II Infinitesimal stability implies stability, Ann. of Math. 89 (1969), 254-291. MR 41:4582

[NPT] Newhouse, S., Palis, J. and Takens, F., Bifurcation and stability of families of diffeomorphisms, Publ. Math. IHES 57 (1983), 5-71. MR 84g:58080

[PT] Palis, J. and Takens, F., Stability of parametrized families of gradient vector fields, Annals of Math. 118 (1983), 383-42. MR 85i:58093

[R] Roussarie, R., Modèles locaux de champs et de formes, Astérisque 30 (1975), 1-181. MR 55: 13444

[T] Takens, F., Partially hyperbolic fixed points, Topology 10 (1971), 133-147. MR 46:6399

Limburgs Universitair Centrum, B-3590 Diepenbeek, Belgium

E-mail address: pbonckae@luc.ac.be 